\title{
Acta
Biochimica
Polonica
}

Vol. 48 No. $3 / 2001$

$719-728$

QUARTERLY

We dedicate this publication to the memory of Professor Jacek Augustyniak

\section{The influence of depletion of voltage dependent anion selective channel on protein import into the yeast Saccharomyces cerevisiae mitochondria}

\author{
Anna Szczechowicz, Lilla Hryniewiecka and Hanna Kmita ${ }^{\bowtie}$
}

Institute of Molecular Biology and Biotechnology, Department of Bioenergetics, Poznań University, Poznań, Poland

Received: 22 January, 2001; revised:15 April, 2001; accepted: 8 March 2001

Key words: mitochondria, protein import, yeast voltage dependent anion selective channel (YVDAC1), YVDAC1-depleted mutants

The supply of substrates to the respiratory chain as well as of other metabolites (e.g. ATP) into inner compartments of mitochondria is crucial to preprotein import into these organelles. Transport of the compounds across the outer mitochondrial membrane is enabled by mitochondrial porin, also known as the voltage-dependent anion-selective channel (VDAC). Our previous studies led to the conclusion that the transport of metabolites through the outer membrane of the yeast Saccharomyces cerevisiae mitochondria missing VDAC (now termed YVDAC1) is considerably restricted. Therefore we expected that depletion of YVDAC1 should also hamper protein import into the mutant mitochondria. We report here that YVDAC1-depleted mitochondria are able to import a fusion protein termed pSu9-DHFR in the amount comparable to that of wild type mitochondria, although over a considerably longer time. The rate of import of the fusion protein into YVDAC1-depleted mitochondria is dis-

The work was supported by a grant from the State Committee for Scientific Research (KBN, Poland) 6PO4A02216).

${ }^{\circledR}$ Hanna Kmita, Institute of Molecular Biology and Biotechnology, Department of Bioenergetics, Poznań University, A. Fredry 10, 61-701 Poznań, Poland; phone: (48 61) 829 4532, fax: (48 61) 829 4503, e-mail: kmita@main.amu.edu.pl

Abbreviations: BSA, bovine serum albumin; DHFR, dihydrofolate reductase; PMSF, phenylmethylsulfonyl fluoride; PSC, peptide sensitive channel; pSu9-DHFR, the fusion protein containing the first 69 amino acids of subunit 9 of the mitochondrial $\mathrm{F}_{1} \mathrm{~F}_{\mathrm{o}}-\mathrm{ATPase}$ of Neurospora crassa in front of entire mouse dihydrofolate reductase (DHFR); TIM, translocase of the inner membrane; TOM, translocase of the outer membrane; $\mathrm{TPP}^{+}$, tetraphenylphosphonium; VDAC, voltage dependent anion selective channel; YVDAC1, yeast VDAC; YVDAC2, yeast VDAC-like protein. 
tinctly lower than into wild type mitochondria probably due to restricted ATP access to the intermembrane space and is additionally influenced by the way the supporting respiratory substrates are transported through the outer membrane. In the presence of ethanol, diffusing freely through lipid membranes, YVDAC1-depleted mitochondria are able to import the fusion protein at a higher rate than in the presence of external NADH which is, like ATP, transported through the outer membrane by facilitated diffusion. It has been shown that transport of external NADH across the outer membrane of YVDAC1-depleted mitochondria is supported by the protein import machinery, i.e. the TOM complex (Kmita \& Budzińska, 2000, Biochim. Biophys. Acta 1509, 86-94.). Since the TOM complex might also contribute to the permeability of the membrane to ATP, it seems possible that external NADH and ATP as well as the imported preprotein could compete with one another for the passage through the outer membrane in YVDAC1-depleted mitochondria.

Protein import and metabolite transport into mitochondria are decisive factors for the proper functioning of these organelles. They are particularly important during cell growth, although the intensive work of mitochondria in non-dividing cells also requires a permanent supply of new proteins which in turn requires an appropriate energetic feed. Import of proteins into mitochondria would not be possible without specialised protein complexes consisting of multiple components enabling the insertion of preproteins into mitochondrial membranes as well as the translocation into the intermembrane space and the matrix. These complexes are known as translocases of the outer and the inner membranes, the TOM and TIM complexes, respectively [1-3]. Transport of metabolites through both mitochondrial membranes is accomplished by different mechanisms. The inner membrane contains specific metabolite carriers while the major pathway for metabolite passage through the outer membrane is believed to be provided by diffusion pores formed by VDAC (voltage dependent anion selective channel), also known as mitochondrial porin [4-7].

However, it is clear now that the presence of VDAC is not crucial to the energetic and metabolic functioning of the yeast $S$. cerevisiae mitochondria, because yeast cells missing the POR 1 gene coding for VDAC are viable on media containing a non-fermentable carbon source [8-10]. Since there are no differences in the electrophoretic protein pattern of the outer membrane between wild type and porinless mitochondria ([9] and our unpublished results), some other protein(s) of the membrane must substitute for the VDAC function. Results of both electrophysiological [10-12] and functional [13-15] studies of mitochondria isolated from different VDAC-depleted mutants of $S$. cerevisiae point to a channel of a diameter smaller than that of VDAC and cationic selectivity. A new protein identified quite recently in the yeast $S$. cerevisiae, designated YVDAC2 because of a $49 \%$ aminoacids sequence identity to yeast VDAC (now termed YVDAC1), is devoid of channel activity but can suppress the phenotype resulting from YVDAC1 depletion when overexpressed [10, 14]. So far only two channels distinct from YVDAC1 have been identified in the outer membrane of $S$. cerevisiae mitochondria, namely the peptide sensitive channel (PSC) $[16,17]$ and the TOM complex channel formed mainly by Tom40 (a membrane-embedded component of the complex) [18-20]. By a combination of biochemical and electrophysiological methods, it was shown that both the TOM complex channel and the PSC serve as preprotein-conducting channels of the outer membrane of mitochondria [18, 19, 21]. Moreover, since the ion-conducting properties of the purified TOM complex resemble those of the PSC, these channels are suggested to be identical [18, 19, 22]. Further, the electophysiological characteristics of both the TOM complex channel and the PSC correspond to that of the channel described by 
Dihanich et al. [11] in the outer mitochondrial membrane of an YVDAC1-depleted $S$. cerevisiae mutant. Thus, transport of metabolites across the outer membrane in YVDAC1-depleted mitochondria can be supported by the TOM complex channel [23, 24]. In this case, preproteins and metabolites would use the same pathway to cross the outer membrane.

Here we demonstrate that a lack of YVDAC1 does not inhibit import of a preprotein termed pSu9-DHFR into the yeast $S$. cerevisiae mitochondria, although the process proceeds at a distinctly lower rate than with wild type mitochondria. Moreover, in the absence of YVDAC1, the transport of a supporting respiratory substrate across the mitochondrial outer membrane and the access of ATP are shown to be essential to the efficiency of the import process.

\section{MATERIALS AND METHODS}

Isolation of mitochondria and mitoplasts. The wild type $S$. cerevisiae strain DBY747 (Mato, ura3-52, leu2-3, leu2-112, his3- $\triangle 1$, trp 1-289) and porin-depleted mutant B5, derived from the above parent strain $[8$, 25], were grown at $28^{\circ} \mathrm{C}$ in YPG medium ( $1 \%$ yeast extract, $2 \%$ peptone, $3 \%$ glycerol), at $\mathrm{pH}$ 5.5. Growth was monitored by determining the absorbance $(A)$ at $546 \mathrm{~nm}$ and the cells were collected in log phase, at the $A$ of about 1.0. Mitochondria were isolated according to published procedure [26]. Mitoplasts were obtained by the swelling-shrinking procedure [26]. The swelling buffer contained $20 \mathrm{mM}$ Hepes, $\mathrm{pH} 6.9$, and $0.2 \%$ bovine serum albumin (BSA). The shrinking buffer contained $1.8 \mathrm{M}$ mannitol, $4 \mathrm{mM} \mathrm{MgCl} 2$ and $0.2 \%$ BSA. The integrity of the outer mitochondrial membrane was monitored by the permeability of the membrane for exogenous cytochrome $c$ [27] or by immunodecoration with antisera against marker proteins of the intermembrane space, cytochrome $c_{1}$ heme lyase and of the mitochondrial matrix, Mge1p (The antisera were kind gifts of Prof. W. Neupert).

In vitro import of a preprotein into isolated mitochondria. A fusion protein, termed pSu9-DHFR, was used as a preprotein for in vitro import assays. The fusion protein contains the first 69 amino-acid residues of subunit 9 of the mitochondrial $\mathrm{F}_{1} \mathrm{~F}_{\mathrm{o}}$-ATPase (pSu9) Neurospora crassa in front of entire mouse dihydrofolate reductase (DHFR) and contains cleavage sites for matrix processing peptidase after residues 35 and 66 [28]. The fusion protein was synthesized in rabbit reticulocyte lysate (Promega) in the presence of $\left[{ }^{35} \mathrm{~S}\right]$ methionine after in vitro transcription by SP6 polymerase from the transcription vector pGEM3 containing the gene for pSu9-DHFR (the $E$. coli strain expressing pSu9-DHFR was a kind gift of Prof. W. Neupert).

Mitochondria were incubated in import buffer (0.6 M mannitol, $50 \mathrm{mM}$ Hepes, $\mathrm{pH} 6.9$, $50 \mathrm{mM} \mathrm{KCl}, 10 \mathrm{mM} \mathrm{MgCl} 2,2 \mathrm{mM} \mathrm{K \textrm {K } _ { 2 }} \mathrm{PO}_{4}$, $2.5 \mathrm{mM}$ EDTA, $0.1 \%$ BSA, $2 \mathrm{mM} \mathrm{NADH}$ or 20 $\mathrm{mM}$ ethanol, with or without $2 \mathrm{mM}$ ATP) at $25^{\circ} \mathrm{C}$ in a final volume of $100 \mu$ l. Reticulocyte lysate containing the radiolabeled preprotein was then added and incubated with mitochondria for the desired times at $25^{\circ} \mathrm{C}$. To assay for pSu9-DHFR import into mitochondria, samples (mitochondria or mitoplasts obtained from mitochondria after the import reaction) were treated with proteinase $\mathrm{K}(50 \mu \mathrm{g} / \mathrm{ml})$ for $10 \mathrm{~min}$ at $0^{\circ} \mathrm{C}$; the enzyme was inactivated by $1 \mathrm{mM}$ phenylmethylsulfonyl fluoride (PMSF). After addition of $900 \mu \mathrm{l}$ of washing buffer ( 0.6 M mannitol, $50 \mathrm{mM}$ Hepes, $\mathrm{pH} 6.9,1 \mathrm{mM}$ PMSF) and reisolation of mitochondria (10 min at $12000 \times \mathbf{g}$ ), pellets were subjected to SDS/PAGE [29]. Accumulation of the mature form of pSu9-DHFR was visualized by fluorography and quantified by Scan Pack 3 .

Other methods. Protein concentrations were measured by the method of Bradford. Respiration of mitochondria and mitoplasts was monitored at $25^{\circ} \mathrm{C}$ with Rank oxygen electrode in an incubation volume of $0.5 \mathrm{ml}$. 
Changes of the inner membrane potential $(\Delta \psi)$ were monitored with a tetraphenylphosphonium $\left(\mathrm{TPP}^{+}\right)$-specific electrode as described in [30]. The values of respiratory control index were calculated according to [31], referring to the resting state following or preceding the phosphorylating state triggered by a given concentration of ADP [15].

\section{RESULTS}

To study the influence of the lack of YVDAC1 on mitochondrial protein import efficiency we used a fusion protein called pSu9-DHFR and studied accumulation of its mature form in the mitochondria of wild type and YVDAC1-depleted strains. Since the employed fusion protein consists of the presequence part of subunit 9 of the $\mathrm{F}_{1} \mathrm{~F}_{\mathrm{o}}$-ATPase (from Neurospora crassa) connected to mouse dihydrofolate reductase, it should be targeted into the mitochondrial ma- electrode - not shown), pSu9-DHFR (p) was not imported into both types of mitochondria, as it could be digested by externally added proteinase $\mathrm{K}(\mathrm{PK})$. However, in the presence of external NADH or ethanol, the fusion protein was efficiently imported into wild type and YVDAC1-depleted mitochondria. A fraction of pSu9-DHFR was processed into the external proteinase $\mathrm{K}$ resistant mature form (m), which indicates location of the mature form inside mitochondria. To check whether the mature form of pSu9-DHFR was indeed located in the mitochondrial matrix we checked its sensitivity to externally added proteinase $\mathrm{K}$ in mitoplasts prepared from mitochondria by selective rupture of the outer membrane after the import reaction (not shown). The absence of the outer membrane allows proteinase $\mathrm{K}$ to digest proteins in the intermembrane space while those located in the matrix are protected. The removal of the outer membrane did not change the accessibility of the externally added protease to the mature

\section{Wild Type}

Mutant

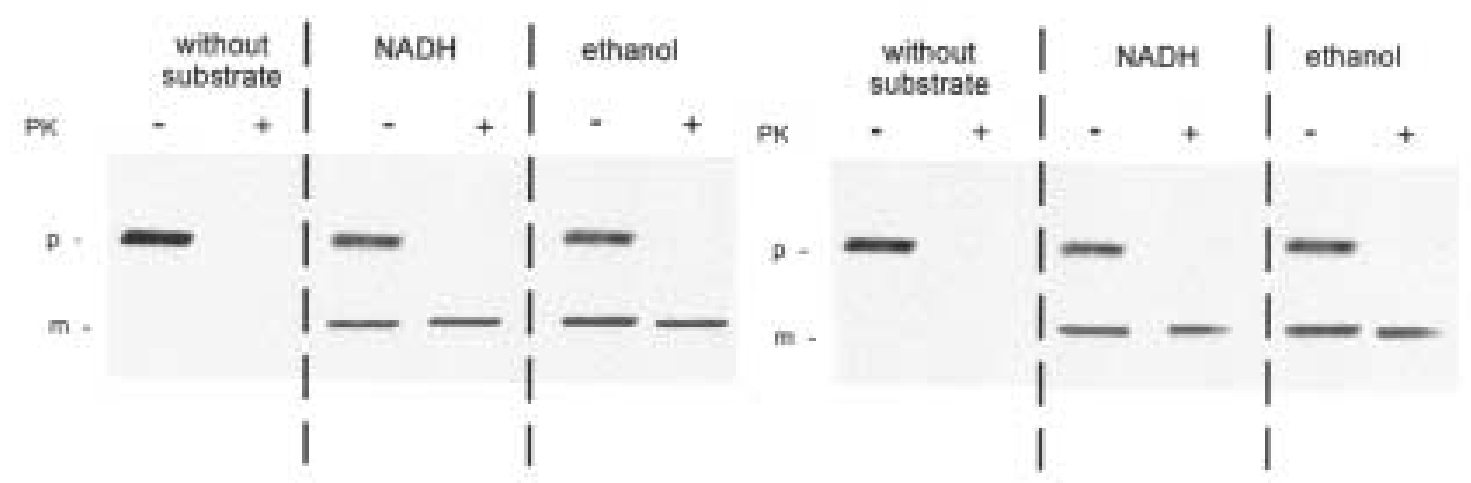

Figure 1. Import of the fusion protein pSu9-DHFR into wild type and YVDAC1-depleted mitochondria.

pSu9-DHFR was imported into yeast mitochondria for $20 \mathrm{~min}$ at $25^{\circ} \mathrm{C}$ in the absence of respiratory substrate or in the presence of $2 \mathrm{mM} \mathrm{NADH}$ or $20 \mathrm{mM}$ ethanol (see Materials and Methods). The import buffer contained $2 \mathrm{mM}$ ATP. After the import reaction each sample was divided in two and one half was treated with $50 \mu \mathrm{g} / \mathrm{ml} \mathrm{proteinase} \mathrm{K}$ for $10 \mathrm{~min}$ at $0^{\circ} \mathrm{C}(+\mathrm{PK})$ while the second one was left untreated (-PK). Next, reisolated mitochondria (10 min, $12000 \times \boldsymbol{g}$ ) were analysed by SDS/PAGE and fluorography.p, pSu9-DHFR; $m$, the mature form of pSu9-DHFR.

trix and simultaneously processed into the mature form devoid of the cleavable presequence [28]. As shown in Fig. 1, in the absence of a respiratory substrate, i.e. in the absence of $\Delta \psi$ (as measured by $\mathrm{TPP}^{+}$-specific form of pSu9-DHFR (see Fig. 1) indicating that in both wild type and YVDAC1-depleted mutant mitochondria, the fusion protein was efficiently targeted into the mitochondrial matrix. 
Next we investigated whether the way of the applied respiratory substrate transport across the outer membrane could affect the import of pSu9-DHFR into wild type and YVDAC1-depleted mitochondria. Both ethanol and external NADH are efficiently oxidised by $S$. cerevisiae mitochondria, but differ by the means they cross the outer membrane. Ethanol diffuses freely through lipid membranes while external $\mathrm{NADH}$ requires a protein (or proteins) in the outer membrane to reach the proper dehydrogenase in the inner membrane, facing the intermembrane space [32]. It should be emphasized here that the rate of oxygen uptake and the levels of the transmembrane potential in the presence of external NADH were quite similar in wild type and YVDAC1-depleted mitochondria and closely comparable to those supported by ethanol (see also [13]). Since YVDAC1 is regarded as the major route for external $\mathrm{NADH}$ through the outer membrane in the S. cerevisiae mitochondria [14, 24], we expected that depletion of YVDAC1 should profoundly hamper the import of pSu9-DHFR in the presence of external NADH. However, as shown in Fig. 1, even in the absence of YVDAC1, both ethanol and external NADH supported the import of pSu9-DHFR into mitochondria. Nevertheless, in YVDAC1-depleted mitochondria, higher amounts of the imported mature form of pSu9-DHFR were obtained in the presence of ethanol, whereas in wild type mitochondria the process proceeded more efficiently in the presence of external NADH, as measured by the external protease-resistant levels of radioactivity accumulated within mitochondrial matrix after a 20 min import reaction (Fig. 1). Thus the lack of YVDAC1 decreased the efficiency of external NADH as a respiratory substrate supporting pSu9-DHFR import into mitochondria.

The weaker import of pSu9-DHFR into YVDAC1-depleted mitochondria in the presence of external NADH than in the presence of ethanol could be due to lower rate of the process caused by the highly reduced perme- ability of the outer membrane for external $\mathrm{NADH}$ observed for the mutant mitochondria $[14,15]$. In such a case the levels of the mature form of pSu9-DHFR imported in the presence of external NADH should exceed those observed in the presence of ethanol if the import reaction is performed sufficiently long. To test this experimentally, we studied the time course of pSu9-DHFR import into wild type and YVDAC1-depleted mitochondria. As shown in Fig. 2, with wild type mitochondria the process proceeded with the same rate for ethanol and external NADH, and the saturation was achieved within approximately 20 min. However, in the presence of external $\mathrm{NADH}$, higher amounts of pSu9-DHFR were imported into the mitochondria. In the case of YVDAC1-depleted mitochondria, the import of pSu9-DHFR was distinctly slower than into wild type mitochondria, even in the presence of ethanol and the saturation was not obtained even after $40 \mathrm{~min}$. Nevertheless, in the presence of ethanol, a higher rate of the process, resulting in higher levels of the mature form of pSu9-DHFR, were observed when compared with external NADH.

It is well known that ATP is essential for the import of the majority of preproteins. Further, we have already shown that transport of the metabolite is restricted in YVDAC1-depleted yeast mitochondria compared to wild type ones as measured by the activity of adenylate kinase in the presence of ATP and AMP [13]. Therefore, it was important to determine whether the presence of ATP was also crucial to the import of $\mathrm{pSu} 9-\mathrm{DHFR}$. As shown in Fig. 3, in the absence of ATP and in the presence of external NADH applied as a respiratory substrate, pSu9-DHFR was only bound with wild type mitochondria at the outer membrane where it was digested by proteinase $\mathrm{K}$, but its processing into the mature form and import did not occur. The same result was obtained with wild type mitochondria in the presence of ethanol and with YVDAC1-depleted mitochondria in the presence of either external NADH or ethanol (not shown). There- 
fore, we concluded that the lower rate of pSu9-DHFR import into YVDAC1-depleted mitochondria was also due to the restricted access of ATP into the intermembrane space. However, both external NADH and ATP could pleted strain studied, described by Michejda et al. [13, 25, 33].

However, both types of mitochondria, i.e. those containing and missing YVDAC1, display different efficiency of pSu9-DHFR im-

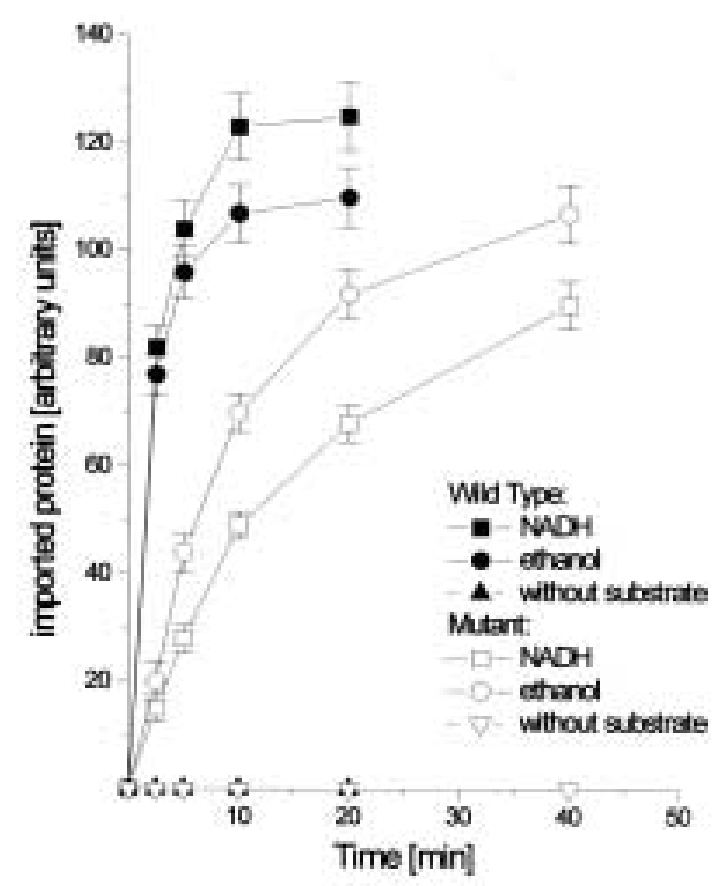

Figure 2. Kinetic analysis of pSu9DHFR import into wild type and YVDAC1-depleted mitochondria.

Isolated mitochondria and pSu9-DHFR were incubated in the import buffer containing $2 \mathrm{mM}$ ATP (see Materials and Methods) in the absence of respiratory substrate or in the presence of $2 \mathrm{mM}$ $\mathrm{NADH}$ or $20 \mathrm{mM}$ ethanol. At the indicated times at $25^{\circ} \mathrm{C}$, samples were withdrawn, chilled on ice and treated with $50 \mu \mathrm{g} / \mathrm{ml}$ proteinase $\mathrm{K}$ for $10 \mathrm{~min}$ at $0^{\circ} \mathrm{C}$. After reisolation of mitochondria all samples were analysed by SDS/PAGE and fluorography. The processed mature form of pSu9-DHFR was quantified by Scan Pack 3. be transported quite efficiently through the outer mitochondrial membrane depleted of YVDAC1 and supported pSu9-DHFR import into the mitochondria.

\section{DISCUSSION}

Effective translocation of the studied preprotein, pSu9-DHFR, into the mitochondrial matrix and its simultaneous processing to the mature form indicate that YVDAC1-depleted mitochondria, like wild type ones, are able to generate an energetic feed sufficient for the import reaction. Thus, the applied respiratory substrates (ethanol or external NADH) enable generation of appropriate levels of the transmembrane potential of the inner membrane $(\Delta \psi)$ and an efficient exchange of adenine nucleotides occurs through both mitochondrial membranes. These conclusions are in agreement with the bioenergetic characteristics of mitochondria of the YVDAC1-de- port, dependent on the respiratory substrate used. Within the applied duration of the import reaction, a higher amount of the mature form of pSu9-DHFR is observed with wild type mitochondria in the presence of external $\mathrm{NADH}$, while in YVDAC1-depleted mitochondria the import of pSu9-DHFR proceeds with a higher efficiency in the presence of ethanol. Further, with wild type mitochondria the same rate of pSu9-DHFR import is observed in the presence of ethanol and external $\mathrm{NADH}$, while with YVDAC1-depleted mitochondria the process is distinctly slower in the presence of external NADH. Simultaneously, a kinetic analysis of the import of pSu9-DHFR into wild type and YVDC1-depleted mitochondria demonstrates that even in the presence of ethanol, diffusing freely through the lipid membranes, YVDAC1-depleted mitochondria import the studied preprotein with a remarkably lower rate. This indicates that depletion of YVDAC1 not only restricts the access of external NADH to the respiratory chain $[14,15]$, 
but also hampers the transport through the outer membrane of other metabolites important for the import competence of mitochondria. For example ATP which is essential for the import of pSu9-DHFR into yeast wild type and YVDAC1-depleted mitochondria.

Taking these data into account one could conclude that the import of pSu9-DHFR into YVDAC1-depleted mitochondria in the presence of external NADH is additionally delayed due to restricted access of the substrate and ATP to the intermembrane space and subsequent competition between them for the en-

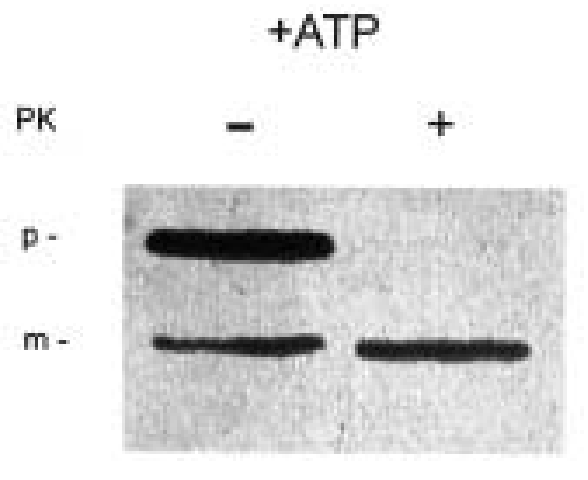

restrictions in the access of external NADH to the intermembrane space of the mitochondria.

We have reported recently that in YVDAC1-depleted mitochondria, the transport of external NADH across the outer membrane is supported by the TOM complex [24]. The TOM complex is known to contain a high-conductance voltage-gated cation-selective channel formed mainly by Tom40 [18, 19, 20]. The channel is thought to be identical to the PSC [18, 19, 22] and to the channel described by Dihanich et al. for another

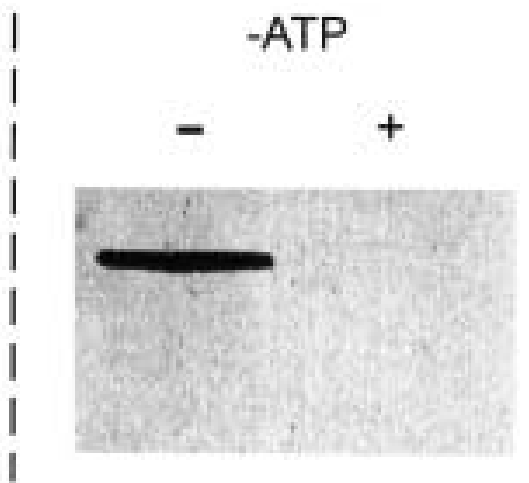

Figure 3. Import of pSu9-DHFR into wild type mitochondria in the presence or absence of ATP.

Isolated mitochondria were incubated with pSu9-DHFR in the import buffer (see Materials and Methods) in the presence of $2 \mathrm{mM} \mathrm{NADH}$ as the respiratory substrate. ATP was omitted in the incubation (-ATP) or applied at the final concentration of $2 \mathrm{mM}$ (+ATP). After the import reaction $\left(20 \mathrm{~min}, 25^{\circ} \mathrm{C}\right.$ ) samples were divided in two and one half was treated with proteinase $\mathrm{K}(50 \mu \mathrm{g} / \mathrm{ml})$ for $10 \mathrm{~min}$ at $0^{\circ} \mathrm{C}(+\mathrm{PK})$ while the second one was left untreated (-PK). Next, mitochondria were reisolated and all samples were analysed by SDS/PAGE and fluorography. p, pSu9-DHFR; $\mathrm{m}$, the mature form of pSu9-DHFR.

try. However, we have reported recently that in the presence of $\Delta \psi$, external $\mathrm{NADH}$ seems to have no effect on the access of $\mathrm{ADP}$ and carboxyatractylate (CATR) into yeast YVDAC1-depleted mitochondria, whereas both ADP and CATR impose constraints on external NADH accessibility [15]. The molecular mass of ATP is comparable to that of ADP but its electrical charge (a negative one) is comparable to that of CATR. Further, oxidation of external NADH by coupled YVDAC1-depleted mitochondria is decreased in the presence of ATP (unpublished results). Therefore, it can be concluded that restricted transport of ATP across the outer membrane of YVDAC1-depleted mitochondria increases
YVDAC1-depleted $S$. cerevisiae mutant [11, 12], and their characteristics correspond to the putative channel proposed for YVDAC1-depleted mutant being the object of the present study [15]. If the TOM complex serves as a rescue channel in YVDAC1-depleted mitochondria, other metabolites required for the import reaction, e.g. ATP, could use the pathway to cross the outer membrane. The differences in the rate of pSu9-DHFR import observed with YVDAC1-depleted mitochondria in the presence of external NADH and ethanol seem to confirm the involvement of the TOM complex in ATP transport across the outer membrane depleted of YVDAC1. In this case both the import of the preprotein 
and the transport of the supporting respiratory substrate and ATP, using the same pathway across the outer membrane, would be additionally restricted in YVDAC1-depleted mitochondria. Thus, external NADH and ATP as well as an imported preprotein could compete for the passage through the outer membrane in YVDAC1-depleted mitochondria. Therefore, it seems possible that the lower rate of pSu9-DHFR import observed with YVDAC1depleted mitochondria in the presence of external NADH results from restricted transport of external NADH to the respiratory chain and of ATP to the intermembrane space as well as of pSu9-DHFR to the TIM complex, i.e. the protein import machinery located in the mitochondrial inner membrane.

The authors wish to thank Prof. W. Neupert for $E$. coli strain and antisera. The technical assistance of D. Drachal-Chrul is gratefully acknowledged.

\section{R E F E R E N C ES}

1. Lill, R. \& Neupert, W. (1996) Mechanisms of protein import across the mitochondrial outer membrane. Trends Cell Biol. 6, 56-61.

2. Pfanner, N. \& Meijer, M. (1997) The Tom and Tim machine. Curr. Biol. 7, R100-R103.

3. Pfanner, N. (1998) Crossing the aqueous intermembrane space. Curr. Biol. 8, R262-R265.

4. Schein, S.J., Colombini, M. \& Finkelstein, A. (1976) Reconstitution in planar lipid bilayers of a voltage-dependent anion-selective channel. J. Membr. Biol. 30, 99-120.

5. Colombini, M. (1979) A candidate for the permeability pathway of the outer mitochondrial membrane. Nature 279, 643-645.

6. Benz, R. (1994) Permeation of hydrophilic solutes through mitochondrial outer membranes:
Review on mitochondrial porins. Biochim. Biophys. Acta 1197, 167-196.

7. Colombini, M., Blachly-Dyson, E. \& Forte, M. (1996) VDAC, a channel in the outer mitochondrial membrane; in Ion Channels (Narahashi, T., ed.) vol. 4, pp. 169-201, Plenum Press, New York.

8. Guo, X.J. \& Lauquin, G.J.-M. (1986) Mitochondrial porin-deficient mutant of $S$. cerevisiae: $I n$ vitro construction and preliminary properties. EBEC Reports 4, 292.

9. Dihanich, M., Suda, K. \& Schatz, G. (1987) A yeast mutant lacking mitochondrial porin is respiratory-deficient, but can recover respiration with simultaneous accumulation of an $86 \mathrm{kD}$ extramitochondrial protein. EMBO J. 6, 723- 728 .

10. Blachly-Dyson, E., Song, J., Wolfgand, W.J., Colombini, M. \& Forte, M. (1997) Multicopy suppressors of phenotype resulting from the absence of yeast VDAC encode a VDAC-like protein. Mol. Cell. Biol. 17, 5727-5738.

11. Dihanich, M., Schmid, A., Oppliger, W. \& Benz, R. (1989) Identification of a new pore in the mitochondrial outer membrane of a porindeficient yeast mutant. Eur. J. Biochem. 181, 703-708.

12. Benz, R., Schmid, A. \& Dihanich, M. (1989) Pores from mitochondrial outer membrane of yeast and a porin-deficient yeast mutant: A comparison. J. Bioener. Biomembr. 21, 439450.

13. Michejda, J., Kmita, H., Stobienia, O., Budzińska, M. \& Lauquin, G.J.-M. (1994) Restriction of metabolite permeation through the outer mitochondrial membrane of porin-deficient yeast mutant; in Molecular Biology of Mitochondrial Transport System (Forte, M. \& Colombini, M., eds.) pp. 341-356, Springer-Verlag, Heidelberg.

14. Lee, A.C., Xu, X., Blachly-Dyson, E., Forte, M. \& Colombini, M. (1998) The role of yeast VDAC genes on the permeability of the mito- 
chondrial outer membrane. J. Membr. Biol. 161, 173-181.

15. Kmita, H., Stobienia, O. \& Michejda, J. (1999) The access of metabolites into yeast mitochondria in the presence and absence of the voltage dependent anion selective channel (YVDAC1). Acta Biochim. Polon. 46, 991-1000.

16. Thieffry, M., Chich, J.M., Goldschmidt, D. \& Henry, J.P. (1988) Incorporation in lipid bilayers of a large conductance cationic channel from mitochondrial membranes. EMBO J. 7, 1449-1454.

17. Fevre, F., Chich, J.F., Lauquin, G.J.-M., Henry, J.P. \& Thieffry, M. (1990) Comparison of mitochondrial cationic channels in wildtype and porin-deficient mutant yeast. FEBS Lett. 262, 201-204.

18. Künkele, K.-P., Heins, S., Dembowski, M., Nargang, F.E., Benz, R., Thieffry, M., Walz, J., Lill, R., Nussberger, S. \& Neupert, W. (1998) The preprotein translocation channel of the outer membrane of mitochondria. Cell 93, 1009-1019.

19. Künkele, K.-P., Juin, P., Pompa, C., Nargang, F.E., Henry, J.-P., Neupert, W., Lill, R. \& Thieffry, M. (1998) The isolated complex of the translocase of the outer membrane of mitoch ondria. J. Biol. Chem. 273, 31032-31039.

20. Hill, K., Model, K., Ryan, M.T., Dietmeier, K., Martin, F., Wagner, R. \& Pfanner, N. (1998) Tom40 forms the hydrophilic channel of the mitochondrial import pore for preproteins. Nature 395, 516-521.

21. Vallette, F.M., Juin, P., Pelleschi, M. \& Henry, J.-P. (1994) Basic peptides can be imported into yeast mitochondria by two distinct pathways. J. Biol. Chem. 269, 13367-13374.

22. Juin, P., Thieffry, M., Henry, J.-P. \& Vallette, F.M. (1997) Relationship between the peptide-sensitive channel and the mitochondrial outer membrane protein translocation machinery. J. Biol. Chem. 272, 6044-6050.
23. Kmita, H., Budzińska, M. \& Michejda, J. (1999) NADH and proteins may use the same pathway to cross the outer membrane in VDAC-depleted mitochondria. Biol. Bull. Poznan 36, 5-15.

24. Kmita, H. \& Budzińska, M. (2000) Involvement of the TOM complex in external NADH transport into yeast mitochondria depleted of porin 1. Biochim. Biophys. Acta 1509, 86-94.

25. Michejda, J., Guo, X.J. \& Lauquin, G.J.-M. (1989) Bioenergetic consequences of the lack of mitochondrial porin: Identification of a putative new pore; in Anion Carriers of Mitochondrial Membranes (Azzi, A., Nałęcz, K.A., Nałęcz, M.J. \& Wojtczak, L., eds.) pp. 225-235, Springer-Verlag, Berlin, Heidelberg.

26. Daum, G., Bohni, P.C. \& Schatz, G. (1982) Import of proteins into mitochondria. Cytochrome $b_{2}$ and cytochrome $c$ peroxidase are located in the intermembrane space of yeast mitochondria. J. Biol. Chem. 257, 13028-13033.

27. Douce, R., Bourguignon, J., Brouquisse, R. \& Neuberger, M. (1984) Isolation of plant mitochondria. General principles and criteria of integrity. Methods Enzymol. 148, 403-415.

28. Pfanner, N., Müller, H.K., Harmey, M.A. \& Neupert, W. (1987) Mitochondrial protein import: Involvement of the mature part of a cleavable precursor protein in the binding to receptor sites. EMBO J. 6, 3449-3454.

29. Laemmli, U.K. (1970) Cleavage of structural proteins during the assembly of the head of bacteriophage T4. Nature 227, 680-685.

30.Kamo, N., Maratsugu, M., Hongoh, R. \& Kobatake, Y.J. (1979) Membrane potential of mitochondria measured with an electrode sensitive to tetraphenyl phosphonium and relationship between proton electrochemical potential and phosphorylation potential in steady state. J. Membr. Biol. 49, 105-121.

31. Chance, B. \& Williams, G.R. (1956) The respiratory chain and oxidative phosphorylation. Adv. Enzymol. 17, 65-87. 
32. de Vries, S. \& Marres, C.A.M. (1991) The mitochondrial respiratory chain of yeast. Structure and biosynthesis and the role in cellular metabolism. Biochim. Biophys. Acta 895, $205-239$.
33. Michejda, J., Guo, X.J. \& Lauquin, G.J.-M. (1990) The respiration of cells and mitochondria of porin deficient yeast mutants is coupled. Biochem. Biophys. Res. Commun. 171, 354-361. 